Review Article

\title{
Invasive and Noninvasive Multimodal Bedside Monitoring in Subarachnoid Hemorrhage: A Review of Techniques and Available Data
}

\author{
Baback Arshi, ${ }^{1}$ William J. Mack, ${ }^{2}$ and Benjamin Emanuel ${ }^{1}$ \\ ${ }^{1}$ Department of Neurology, University of Southern California, Health Sciences Campus, CHP 209 D, Mail Code 9207, Los Angeles, \\ CA 90089-9207, USA \\ ${ }^{2}$ Department of Neurosurgery, University of Southern California, Health Sciences Campus, CHP 209 D, Mail Code 9207, Los Angeles, \\ CA 90089-9207, USA
}

Correspondence should be addressed to Benjamin Emanuel; ben.a.emanuel@gmail.com

Received 5 November 2012; Accepted 9 March 2013

Academic Editor: Jay Mocco

Copyright (c) 2013 Baback Arshi et al. This is an open access article distributed under the Creative Commons Attribution License, which permits unrestricted use, distribution, and reproduction in any medium, provided the original work is properly cited.

Delayed-cerebral ischemia is a major cause of morbidity and mortality in the setting of aneurysmal subarachnoid hemorrhage. Despite extensive research efforts and a breadth of collective clinical experience, accurate diagnosis of vasospasm remains difficult, and effective treatment options are limited. Classically, diagnosis has focused on imaging assessment of the cerebral vasculature. Recently, invasive and noninvasive bedside techniques designed to characterize relevant hemodynamic and metabolic alterations have gained substantial attention. Such modalities include microdialysis, brain tissue oxygenation, jugular bulb oximetry, thermal diffusion cerebral blood flow, and near-infrared spectroscopy. This paper reviews these modalities and examines data pertinent to the diagnosis and management of cerebral vasospasm.

\section{Introduction}

Delayed-cerebral ischemia (DCI) is a major cause of morbidity and mortality in the setting of aneurysmal subarachnoid hemorrhage (aSAH) [1]. Early diagnosis and effective treatment of cerebral vasospasm remain considerable challenges. Neurologists, neurosurgeons, and intensivists utilize multiple advanced diagnostic imaging techniques and examine a broad spectrum of physiologic parameters in attempts to identify reversible cerebral ischemia following aSAH. These modalities provide important data that guide treatment decisions and influence management protocols. Nonetheless, vasospasm related morbidity and mortality remain exceedingly high. Over the past twenty years, efforts have centered on identifying metabolic substrates relevant to the pathogenesis of cerebral vasospasm. As a result, intracerebral monitoring has given rise to a new, multimodal discipline providing specialists, a wide a variety of novel biomarkers potentially relevant to the diagnosis and management of delayed cerebral ischemia. This paper reviews both invasive and noninvasive multimodal bedside monitoring strategies and examines data pertinent to the diagnosis and management of cerebral vasospasm.

\section{Methods}

An extensive literature search through PubMed medical database through July 2012 was conducted using combinations of the keywords "aneurysmal subarachnoid hemorrhage," "vasospasm," "microdialysis," "brain tissue oxygenation (Licox, Integra Neurosciences)," "jugular bulb oximetry," "thermal diffusion cerebral blood flow (Hemedex, Hemedex Inc.)," and "near-infrared spectroscopy." All titles and abstracts identified were reviewed. Additional articles were identified from the reference lists of the selected manuscripts. Articles in all languages were eligible. Inclusion was restricted to reports containing original data from studies examining microdialysis, brain tissue oxygenation, jugular bulb oximetry, thermal diffusion cerebral blood flow, or near infrared 
spectroscopy in the setting of aSAH. Trials incorporating imaging to validate the detection of delayed cerebral ischemia with invasive intracerebral monitoring as well as trials that did not have imaging correlates were included. Articles that lacked original data including review articles, meta-analysis, and case reports were excluded. Trials evaluating multimodal modalities in patients with only traumatic brain injury (TBI) were also excluded.

\section{Results}

3.1. Microdialysis. Invasive microdialysis monitoring enables assessment of regional cerebral metabolic physiology and provides biomarkers for clinical correlation [2]. Extracellular pyruvate $(166 \pm 47 \mu \mathrm{mol} / \mathrm{L})$, lactate $(2.9 \pm 0.9 \mathrm{mmol} / \mathrm{L})$, glucose $(1.7 \pm 0.9 \mathrm{mmol} / \mathrm{L})$, glutamate $(1.6 \pm 1.6 \mu \mathrm{mol} / \mathrm{L})$, glycerol $(82 \pm 44 \mu \mathrm{mol} / \mathrm{L})$, and lactate/pyruvate ratio $(23 \pm 4)$ have been studied, and reference ranges established [3].

Studies have suggested that extracellular alterations in glutamate, lactate, lactate/pyruvate ratio, and glycerol are associated with delayed cerebral ischemia following aSAH [4-7]. Recent attempts have been made to correlate metabolic changes with findings on TCD, computed tomography (CT), and positron emission tomography-computed tomography (PET CT). A 2001 study of sixty patients by Unterberg et al. demonstrated that microdialysis values had a higher specificity $(0.89,95 \%$ CI $0.78-1)$ for delayed cerebral ischemia in symptomatic vasospasm patients than did TCD $(0.63,95 \%$ CI $0.46-0.8)$ or digital subtraction angiography $(0.53,95 \%$ CI $0.35-0.7)$ [8].

A study of forty aSAH patients conducted by Kett-White et al. correlated microdialysis levels with clinical presentation grade. The authors demonstrated that unfavorable World Federation of Neurosurgical Societies (WFNS) scores were associated with higher extracellular glutamate, lactate, and lactate/pyruvate ratio.

Patients presenting with a WFNS grade 1,2 , or 3 demonstrated mean levels of extracellular glutamate $(3.5 \mu \mathrm{mol} / \mathrm{L}$, 95\% CI 2.1-4.9), lactate (2.3 mmol/L, 95\% CI 1.7-2.9), and lactate/pyruvate ratio $(20.8,95 \%$ CI 16.6-25) that were significantly lower than those of WFNS grade 4 or 5 patients ( glutamate $5.5 \mu \mathrm{mol} / \mathrm{L}$; 95\% CI 1.6-9.4; lactate $3.7 \mathrm{mmol} / \mathrm{L}$; 95\% CI 2.9-4.5; and lactate/pyruvate ratio 32.0; 95\% CI 25.738.3) [9]. These findings have been corroborated by several independent studies [4-7].

Studies have also correlated extracellular cerebral microdialysate values with findings on diagnostic imaging. Prior studies have shown that PET can reliably demonstrate reduction in cerebral blood flow secondary to vasospasm [10]. Sarrafzadeh et al. demonstrated that a reduction in regional cerebral blood flow (rCBF), as determined by ${ }^{15} \mathrm{O}-\mathrm{H}_{2} \mathrm{O}$ PET, is best reflected by elevations in extracellular glutamate and glycerol levels [6]. The authors utilized the following microdialysis parameters for detection of cerebral ischemia: glutamate $>5 \mu \mathrm{mol} / \mathrm{L}$, lactate $>4 \mathrm{mmol} / \mathrm{L}$, lactate/pyruvate ratio $>25$, glycerol $>70 \mathrm{mmol} / \mathrm{L}$, and reduced $\mathrm{rCBF}<$ $20 \mathrm{~mL} \cdot 100 \mathrm{~g}^{-1} \cdot \mathrm{min}^{-1}[6]$.
3.2. Brain Tissue Oxygenation. Brain tissue oxygenation $\left(\mathrm{PbtO}_{2}\right)$ catheters quantify brain tissue oxygen pressure in focal cortical regions as a surrogate for alterations in oxygen delivery or cerebral demand [11]. Studies have demonstrated a predisposition for cerebral ischemia when $\mathrm{PbtO}_{2}$ levels were $<10 \mathrm{~mm} \mathrm{Hg}[12,13]$. A large trial conducted by Jaeger et al. evaluated $\mathrm{PbtO}_{2}$ monitoring in the setting of cerebral vasospasm. The authors demonstrated that $\mathrm{PbtO}_{2}$ could accurately predict impaired vascular autoregulation. In a study of sixty-seven patients, there was significant differences in $\mathrm{PbtO}_{2}$ levels between patient cohorts with $(20.8 \mathrm{~mm} \mathrm{Hg} \pm$ 5.0) and without $(23.9 \mathrm{~mm} \mathrm{Hg} \pm 5.8)(P<0.06)$ evidence of cerebral infarction [14]. Of note, the $\mathrm{PbtO}_{2}$ values differ from those reported by Meixensberger et al. and Väth et al., perhaps owing to sampling at earlier posthemorrhage time points including days 1-4 $[12,13]$. Nevertheless, trends indicate that lower $\mathrm{PbtO}_{2}$ is suggestive of cerebral ischemia. In the patients without evidence of infarction on CT scan, maximum MCA velocities were $160 \pm 46 \mathrm{~cm} / \mathrm{s}$, while values in those with evidence of cerebral infarction were $172 \pm 46 \mathrm{~cm} / \mathrm{s}$ $(P=0.32)[14]$.

In a twenty-three-patient study, Cerejo et al. successfully correlated $\mathrm{PbtO}_{2}$ measurements with evidence of vasospasm on TCD $(\mathrm{MCA}>120-180 \mathrm{~cm} / \mathrm{s}$, ICA $>130 \mathrm{~cm} / \mathrm{s}$, ACA > $120 \mathrm{~cm} / \mathrm{s}$, PCA $>90 \mathrm{~cm} / \mathrm{s}$, Basilar $>100 \mathrm{~cm} / \mathrm{s}$, and Vertebral $>90 \mathrm{~cm} / \mathrm{s}$ ) [15]. Postoperative vasospasm patients had significantly lower $\mathrm{PbtO}_{2}$ values of $5.4 \mathrm{~mm} \mathrm{Hg}$ versus $11 \mathrm{~mm} \mathrm{Hg}$ $(P=0.002)$ [15]. These results corroborate findings by Meixensberger et al. suggesting that $\mathrm{PbtO}_{2}<10 \mathrm{~mm} \mathrm{Hg}$ is suggestive of regional cerebral ischemia [12]. The trend demonstrated that cerebral hypoxia was associated with poor outcomes.

A study by Meixensberger et al. sought to utilize intracranial pressure (ICP), cerebral perfusion pressure (CPP), and $\mathrm{PbtO}_{2}$ as complementary tools in the guidance of vasospasm therapy [16]. However, the investigation did not establish early $\mathrm{PbtO}_{2}$ levels as an accurate predictor of outcome [16]. $\mathrm{PbtO}_{2}$ measurements allowed for detection of cerebral infarction, but not prediction of cerebral ischemia. The lack of predictive value rendered $\mathrm{PbtO}_{2}$ less useful than ICP in guiding clinical therapies [16]. Due to sensitivity for symptomatic ischemia, hypertensive, hypervolemic, and hemodilution $(\mathrm{HHH})$ therapy was guided by ICP values, rather than $\mathrm{PbtO}_{2}$. Ramakrishna et al. showed $\mathrm{PbtO}_{2}$ to be higher in aSAH survivors, $33.94 \mathrm{~mm} \mathrm{Hg} \pm 2.74$, when compared to nonsurvivors, $28.14 \mathrm{~mm} \mathrm{Hg} \pm 2.59(P=0.05)$ [17]. The threshold for cerebral hypoxia in this study was $\mathrm{PbtO}_{2}<15 \mathrm{~mm} \mathrm{Hg}$ in this study. Despite a relatively high threshold, compromised $\mathrm{PbtO}_{2}$ correlated with 1-month mortality following $\mathrm{SAH}$ [17].

While data can be extrapolated, and information can be gained from the results of large TBI trials, further studies are required to validate the routine use of $\mathrm{PbtO}_{2}$ in the setting of aSAH.

3.3. Jugular Bulb Oximetry. Jugular bulb oximetry has been used to monitor jugular venous desaturation. It is hypothesized that low jugular venous oxygen levels and anaerobic 
metabolism are associated with poor neurologic outcome [18]. Heran et al. examined the utility of increased cerebral oxygen extraction $\left(\mathrm{AVDO}_{2}\right)$ and resultant jugular venous desaturation, as evidenced by jugular bulb oximetry, in predicting cerebral vasospasm [19]. Of the 14 patients studied, those who developed vasospasm demonstrated changes in jugular venous oxygen saturation. In a matched analysis, the vasospasm cohort had a baseline $\mathrm{AVDO}_{2}$ of $27.6 \%$, while the nonvasospasm patients had an $\mathrm{AVDO}_{2}$ of $40.0 \%$ [19].

3.4. Thermal Diffusion Cerebral Blood Flow. Thermal-diffusion flowmetry utilizes cerebral blood flow measurements to predict vasospasm. Probes are placed in "at-risk" white matter regions through intracranial bolts and are used to measure regional cerebral blood flow [20]. Although most data is generated from TBI patients, a study by Vajkoczy et al. demonstrated utility in the setting of aSAH. In a 14-patient study, Hemedex monitors appeared effective in predicting cerebral vasospasm as defined by angiography, Xe-enhanced CT scans, and clinical parameters [21]. In vasospasm patients, thermal-diffusion flowmetry (TD-rCBF) measurements decreased from $21 \pm 4$ to $9 \pm 1 \mathrm{~mL} / 100 \mathrm{~g} / \mathrm{min}$, while the control group maintained cerebral blood flow values of $25 \pm$ $4 \mathrm{~mL} / 100 \mathrm{~g} / \mathrm{min}$. The authors identified a TD-rCBF value of $15 \mathrm{~mL} / 100 \mathrm{~g} / \mathrm{min}$ as an appropriate threshold for the diagnosis of symptomatic vasospasm [21].

3.5. Near-Infrared Spectroscopy. Near-infrared spectroscopy (NIRS) allows for monitoring of cerebral blood flow by local hemoglobin oxygen saturation $\left(\mathrm{rSO}_{2}\right)$ measurement [22-24]. Probes are noninvasively secured to the scalp with adhesive pads, allowing for prolonged monitoring. The majority of studies to date have employed infrared light at uniform wavelengths of $730 \mathrm{~nm}, 810 \mathrm{~nm}$, and $850 \mathrm{~nm}$ [22-24]. Mutoh et al. continuously monitored patients and compared $\mathrm{rSO}_{2}$ to TCD, MR angiography, and stable technetium-99 m hexamethylpropyleneamine oxime single photon emission computed tomography (SPECT). Decreased $\mathrm{rSO}_{2}$ was associated with cerebral vasospasm according to MCA TCD flow velocities > $120 \mathrm{~cm} / \mathrm{s}$, Lindegaard ratio $>3$, and/or imaging criteria [22]. Specifically, affected patients demonstrated a $24 \% \pm 4 \%$ ipsilateral decrease in $\mathrm{rSO}_{2}$ compared to measurements from the contralateral, unaffected hemisphere [22].

A further study comparing NIRS performance to daily TCD measurements demonstrated early prediction of vasospasm [25]. In fact NIRS showed an abrupt decrease in $\mathrm{rSO}_{2}$ between days 5-9 after aSAH, which correlated with vasospasm on cerebral angiography [25]. Monitoring capability has improved via reductions in hemoglobin concentration variability and adjustments controlling for effects attributable to cerebrospinal fluid and skull thickness [26-28].

\section{Conclusion}

Efforts focused on the application of novel neurointensive monitoring techniques in the setting of cerebral vasospasm have yielded encouraging results. Multiple international sites have contributed to an expanding cache of research that has generated significant interest within the academic community. While these modalities have not yet gained universal acceptance or widespread implementation, they hold real promise for future application. Large-scale prospective trials are needed for validation. Comparative analyses among the proposed modalities would help determine relative effectiveness in a range of clinical settings. Considerable research efforts have focused on Microdialysis, $\mathrm{PbtO}_{2}$, and near-infrared spectroscopy, yet data is less abundant for Jugular bulb oximetry and thermal diffusion cerebral blood flow. While invasive neuromonitoring may possess diagnostic utility in the setting of delayed cerebral vasospasm, additional research is needed to determine the efficacy of the different monitoring modalities and refine our understanding of their clinical applications.

\section{References}

[1] M. R. Mayberg, H. H. Batjer, R. Dacey et al., "Guidelines for the management of aneurysmal subarachnoid hemorrhage: a statement for healthcare professionals from a special writing group of the Stroke Council, American Heart Association," Stroke, vol. 25, no. 11, pp. 2315-2328, 1994.

[2] P. J. Hutchinson, M. T. O’Connell, P. G. Al-Rawi et al., "Clinical cerebral microdialysis: a methodological study," Journal of Neurosurgery, vol. 93, no. 1, pp. 37-43, 2000.

[3] P. Reinstrup, N. Ståhl, P. Mellergård, T. Uski, U. Ungerstedt, and C. H. Nordström, "Intracerebral microdialysis in clinical practice: baseline values for chemical markers during wakefulness, anesthesia, and neurosurgery," Neurosurgery, vol. 47, no. 3, pp. 701-710, 2000.

[4] O. G. Nilsson, L. Brandt, U. Ungerstedt, and H. Säveland, "Bedside detection of brain ischemia using intracerebral microdialysis: subarachnoid hemorrhage and delayed ischemic deterioration," Neurosurgery, vol. 45, no. 5, pp. 1176-1185, 1999.

[5] A. Sarrafzadeh, D. Huax, O. Sakowtz et al., "Acute focal neurological deficits in aneurysmal subarachnoid hemorrhage: relation of clinical course, CT findings, and metabolite abnormalities monitored with bedside microdialysis," Stroke, vol. 34, no. 6, pp. 1382-1388, 2003.

[6] A. S. Sarrafzadeh, D. Haux, L. Lüdemann et al., "Cerebral ischemia in aneurysmal subarachnoid hemorrhage: a correlative microdialysis-PET study," Stroke, vol. 35, no. 3, pp. 638-643, 2004.

[7] A. S. Sarrafzadeh, A. Nagel, M. Czabanka, T. Denecke, P. Vajkoczy, and M. Plotkin, "Imaging of hypoxic-ischemic penumbra with ${ }^{18} \mathrm{~F}$-fluoromisonidazole PET/CT and measurement of related cerebral metabolism in aneurysmal subarachnoid hemorrhage," Journal of Cerebral Blood Flow and Metabolism, vol. 30, no. 1, pp. 36-45, 2010.

[8] A. W. Unterberg, O. W. Sakowitz, A. S. Sarrafzadeh, G. Benndorf, and W. R. Lanksch, "Role of bedside microdialysis in the diagnosis of cerebral vasospasm following aneurysmal subarachnoid hemorrhage," Journal of Neurosurgery, vol. 94, no. 5, pp. 740-749, 2001.

[9] R. Kett-White, P. J. Hutchinson, P. G. Al-Rawi et al., "Adverse cerebral events detected after subarachnoid hemorrhage using brain oxygen and microdialysis probes," Neurosurgery, vol. 50, no. 6, pp. 1213-1222, 2002.

[10] K. D. Yundt, R. L. Grubb Jr., M. N. Diringer, and W. J. Powers, "Autoregulatory vasodilation of parenchymal vessels is 
impaired during cerebral vasospasm," Journal of Cerebral Blood Flow and Metabolism, vol. 18, pp. 419-424, 1998.

[11] E. Maloney-Wilensky and P. Le Roux, "The physiology behind direct brain oxygen monitors and practical aspects of their use," Child's Nervous System, vol. 26, no. 4, pp. 419-430, 2010.

[12] J. Meixensberger, E. Kunze, E. Barcsay, A. Vaeth, and K. Roosen, "Clinical cerebral microdialysis: brain metabolism and brain tissue oxygenation after acute brain injury," Neurological Research, vol. 23, no. 8, pp. 801-806, 2001.

[13] A. Väth, E. Kunze, K. Roosen, and J. Meixensberger, “Therapeutic aspects of brain tissue $\mathrm{pO}_{2}$ monitoring after subarachnoid hemorrhage," Acta Neurochirurgica, no. 81, pp. 307-309, 2002.

[14] M. Jaeger, M. U. Schuhmann, M. Soehle, C. Nagel, and J. Meixensberger, "Continuous monitoring of cerebrovascular autoregulation after subarachnoid hemorrhage by brain tissue oxygen pressure reactivity and its relation to delayed cerebral infarction," Stroke, vol. 38, no. 3, pp. 981-986, 2007.

[15] A. Cerejo, P. A. Silva, A. Vilarinho, C. Dias, and R. Vaz, "Intraoperative brain oxygenation monitoring and vasospasm in aneurysmal subarachnoid hemorrhage," Neurological Research, vol. 34, pp. 181-186, 2012.

[16] J. Meixensberger, A. Vath, M. Jaeger, E. Kunze, J. Dings, and K. Roosen, "Monitoring of brain tissue oxygenation following severe subarachnoid hemorrhage," Neurological Research, vol. 25, no. 5, pp. 445-450, 2003.

[17] R. Ramakrishna, M. Stiefel, J. Udoetuk et al., "Brain oxygen tension and outcome in patients with aneurysmal subarachnoid hemorrhage," Journal of Neurosurgery, vol. 109, pp. 1075-1082, 2008.

[18] W. M. Coplin, G. E. O'Keefe, M. Sean Grady et al., "Thrombotic, infectious, and procedural complications of the jugular bulb catheter in the intensive care unit," Neurosurgery, vol. 41, no. 1, pp. 101-109, 1997.

[19] N. S. Heran, S. J. Hentschel, and B. D. Toyota, "Jugular bulb oximetry for prediction of vasospasm following subarachnoid hemorrhage," Canadian Journal of Neurological Sciences, vol. 31, no. 1, pp. 80-86, 2004.

[20] G. Rosenthal, R. O. Sanchez-Mejia, N. Phan, J. C. Hemphill, C. Martin, and G. T. Manley, "Incorporating a parenchymal thermal diffusion cerebral blood flow probe in bedside assessment of cerebral autoregulation and vasoreactivity in patients with severe traumatic brain injury: clinical article," Journal of Neurosurgery, vol. 114, no. 1, pp. 62-70, 2011.

[21] P. Vajkoczy, P. Horn, C. Thome, E. Munch, and P. Schmiedek, "Regional cerebral blood flow monitoring in the diagnosis of delayed ischemia following aneurysmal subarachnoid hemorrhage," Journal of Neurosurgery, vol. 98, no. 6, pp. 1227-1234, 2003.

[22] T. Mutoh, T. Ishikawa, A. Suzuki, and N. Yasui, "Continuous cardiac output and near-infrared spectroscopy monitoring to assist in management of symptomatic cerebral vasospasm after subarachnoid hemorrhage," Neurocritical Care, vol. 13, no. 3, pp. 331-338, 2010.

[23] C. Zweifel, G. Castellani, M. Czosnyka et al., "Continuous assessment of cerebral autoregulation with near-infrared spectroscopy in adults after subarachnoid hemorrhage," Stroke, vol. 41, no. 9, pp. 1963-1968, 2010.

[24] A. Brawanski, R. Faltermeier, R. D. Rothoerl, and C. Woertgen, "Comparison of near-infrared spectroscopy and tissue $\mathrm{Po}_{2}$ time series in patients after severe head injury and aneurysmal subarachnoid hemorrhage," Journal of Cerebral Blood Flow and Metabolism, vol. 22, no. 5, pp. 605-611, 2002.
[25] N. Yokose, K. Sakatani, Y. Murata et al., "Bedside assessment of cerebral vasospasms after subarachnoid hemorrhage by near infrared time-resolved spectroscopy," Advances in Experimental Medicine and Biology, vol. 662, pp. 505-511, 2010.

[26] K. Yoshitani, M. Kawaguchi, N. Miura et al., "Effects of hemoglobin concentration, skull thickness, and the area of the cerebrospinal fluid layer on near-infrared spectroscopy measurements," Anesthesiology, vol. 106, no. 3, pp. 458-462, 2007.

[27] K. Kishi, M. Kawaguchi, K. Yoshitani, T. Nagahata, and H. Furuya, "Influence of patient variables and sensor location on regional cerebral oxygen saturation measured by INVOS 4100 near-infrared spectrophotometers," Journal of Neurosurgical Anesthesiology, vol. 15, no. 4, pp. 302-306, 2003.

[28] P. G. Al-Rawi, P. Smielewski, and P. J. Kirkpatrick, "Evaluation of a near-infrared spectrometer (NIRO 300) for the detection of intracranial oxygenation changes in the adult head," Stroke, vol. 32, no. 11, pp. 2492-2499, 2001. 


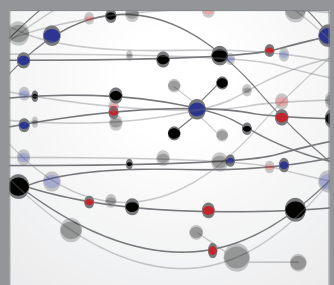

The Scientific World Journal
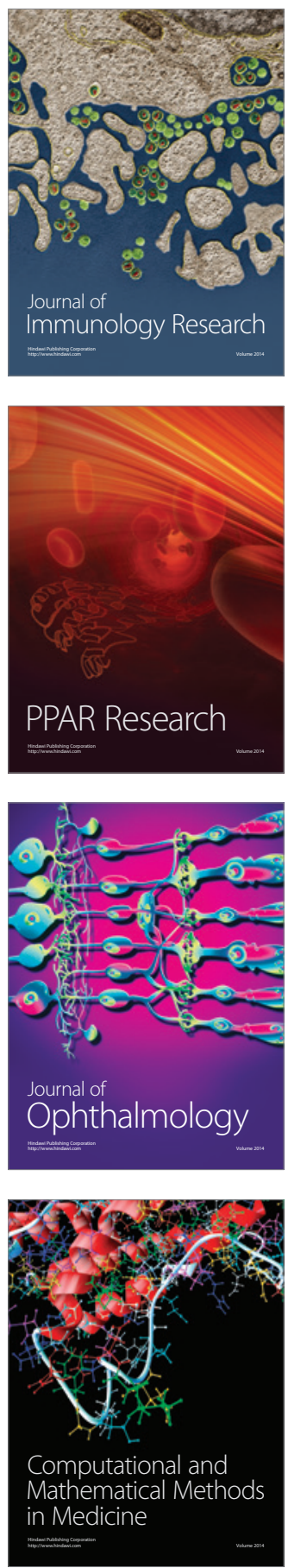

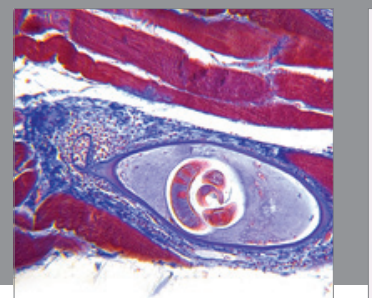

Gastroenterology

Research and Practice
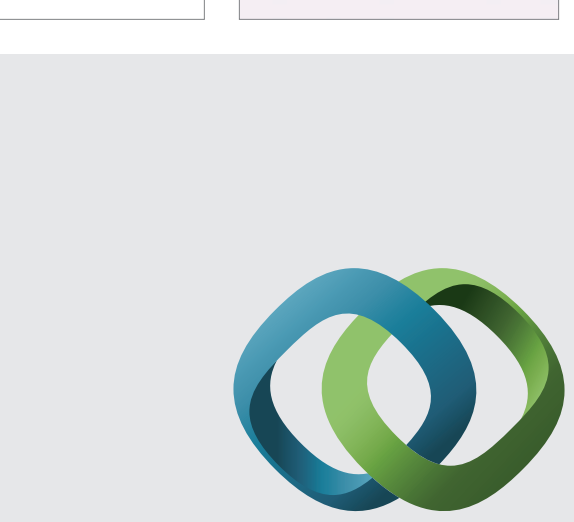

\section{Hindawi}

Submit your manuscripts at

http://www.hindawi.com
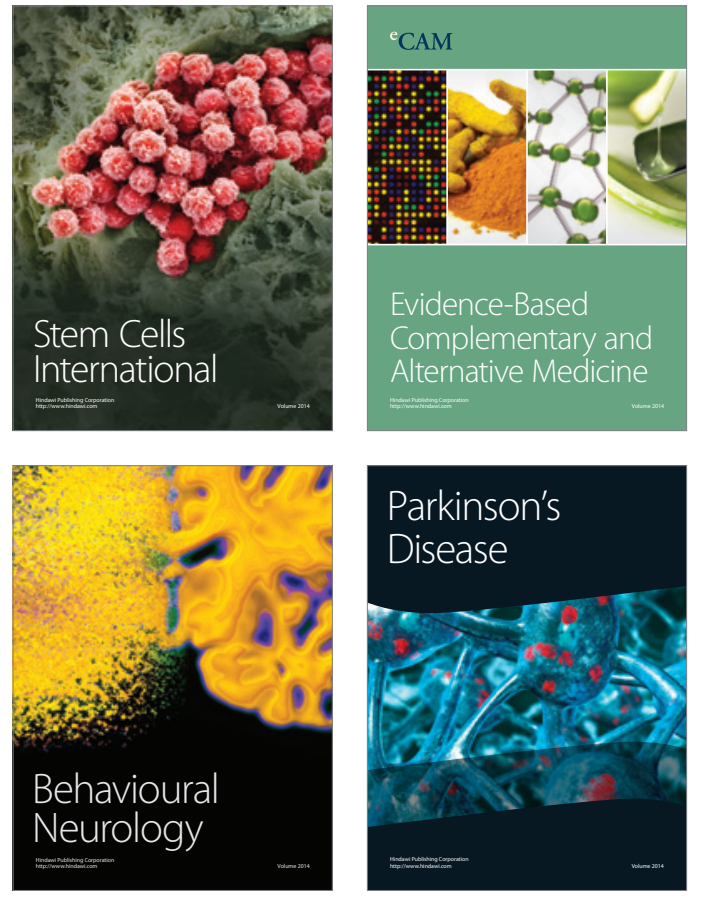
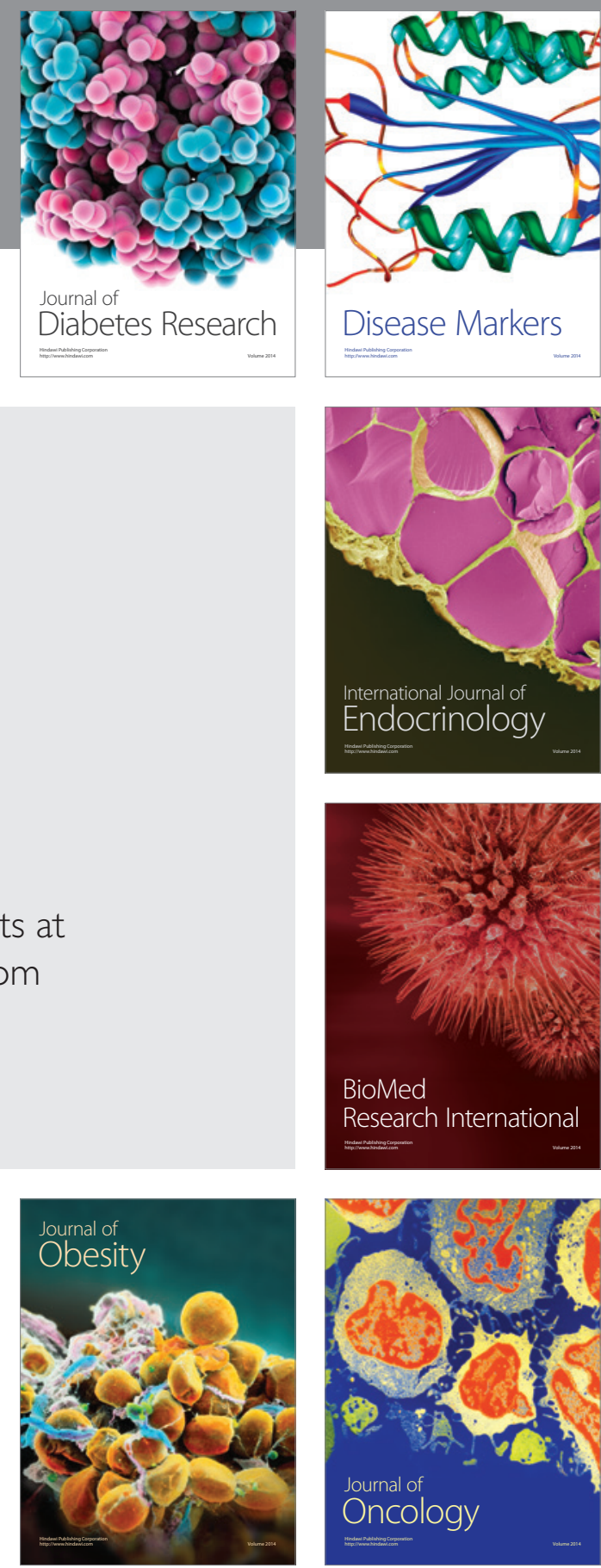

Disease Markers
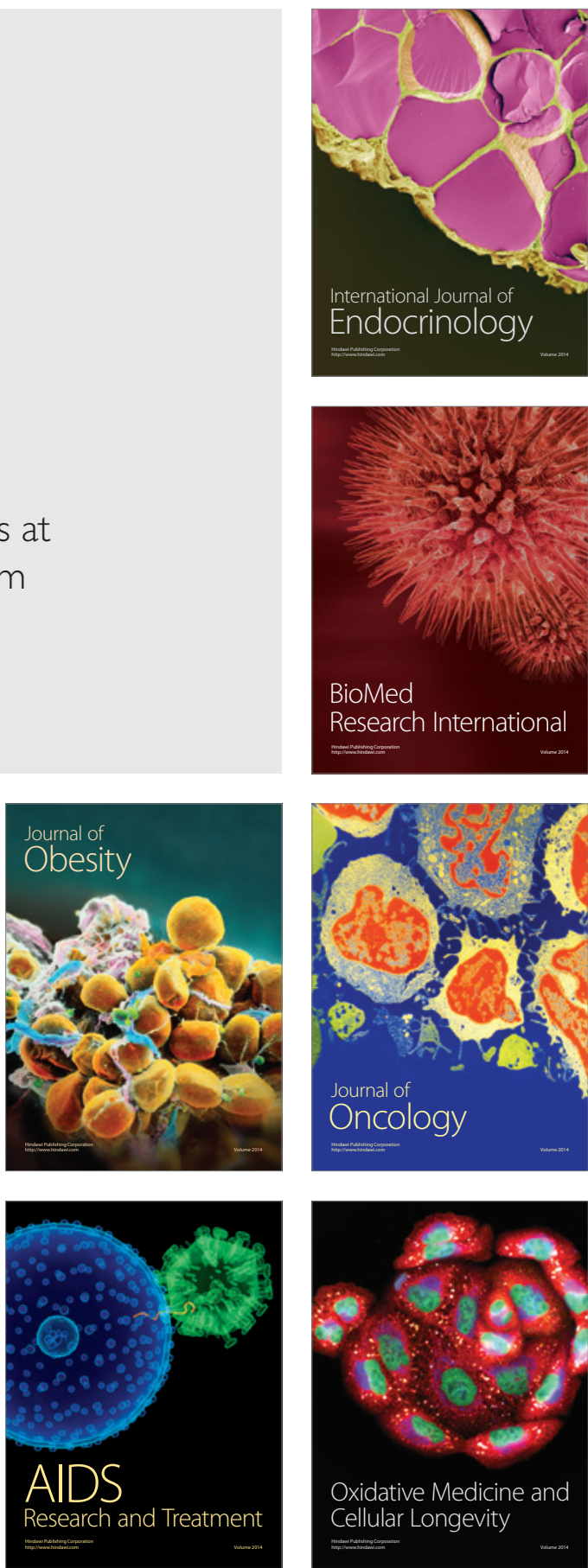